\title{
Using a family of dividing surfaces normal to the minimum energy path for quantum instanton rate constants
}

\author{
Yimin Li and William H. Miller \\ Department of Chemistry and Kenneth S. Pitzer Center for Theoretical Chemistry, \\ University of California, and Chemical Sciences Division, \\ Lawrence Berkeley National Laboratory, Berkeley, California 94720
}

One of the outstanding issues in the quantum instanton (QI) theory (or any transition state-type theory) for thermal rate constants of chemical reactions is the choice of an appropriate 'dividing surface' (DS) that separates reactants and products. (In the general version of the QI theory, there are actually two dividing surfaces involved.) This paper shows one simple and general way for choosing DS's for use in QI Theory, namely using the family of (hyper) planes normal to the minimum energy path (MEP) on the potential energy surface at various distances $s$ along it. Here the reaction coordinate is not one of the dynamical coordinates of the system (which will in general be the Cartesian coordinates of the atoms), but rather simply a parameter which specifies the DS. It is also shown how this idea can be implemented for an $N$ atom system in $3 \mathrm{~d}$ space in a way that preserves overall translational and rotational invariance. Numerical application to a simple system (the collinear $H+H_{2}$ reaction) is presented to illustrate the procedure.

\section{INTRODUCTION}

The specification of a dividing surface (DS) is a critical component of any transition state-like theory for chemical reaction rates. In classical mechanics, for example, transition

state theory $(\mathrm{TST})^{1}$ gives the exactly correct rate if one can find a DS that no trajectories cross more than once. ${ }^{2-4}$ (With any other DS, TST gives a rate constant that is too large.) 
This has been appropriately referred to a Wigner's 'fundamental assumption' for the validity of classical TST. ${ }^{5}$ Choosing the best possible DS is also important for the accuracy of any quantum versions of $\mathrm{TST}^{6}$, though here one does not have the variational principle of classical TST (i.e., that since the TST rate is an upper bound to the correct rate for any DS, the best DS is the one that makes the TST rate a minimum).

A theory of reaction rates recently developed in this research group - referred to as the quantum instanton (QI) model or approximation ${ }^{7,8}$ - has shown a high degree of accuracy both at low temperature, where tunneling effects are large, and also at higher temperature where they are not. The term 'quantum instanton' refers to its relation to an earlier semiclassical (SC) theory ${ }^{9}$ of reaction rates that became known as the instanton model (though the originator referred to it as SC-TST). The essential difference between the QI model and its $\mathrm{SC}$ version is that the rate constant is expressed wholly in terms of the quantum Boltzmann operator rather than its SC approximation. Evaluation of the QI rate expression is thus possible for very complex molecular systems by using Monte Carlo path integral methods to evaluate the Boltzmann operator. ${ }^{10-13}$ Since the QI model (and its earlier SC version) incorporate TST-like approximations, it is rightly viewed as a type of quantum TST (of which there is no unique version, unlike the situation in classical mechanics).

Internal to the QI theory ${ }^{7,8}$ is a variational criterion [not to be confused with the variational principle of classical TST] for choosing a DS (actually two DS's in the low temperature tunneling regime, one on the reactant side, and one on the product side of the transition state region). For systems with many degrees of freedom, however, it is not feasible to vary the form of the DS without limit. The purpose of this paper is to describe a simple but general way of choosing a family of DS's for use in the QI rate theory. A DS is in general specified by setting some function of coordinates equal to 0 ,

$$
\xi(\mathbf{x})=0
$$

where throughout this paper we take the coordinates of the system, $\mathbf{x}$, to be Cartesian. By convention, $\xi(\mathbf{x})$ is negative on the reactant side of the dividing surface, and positive on the 
product side of the dividing surface.

For relatively simple reaction systems, it is common to postulate a DS based on physical intuition. For example, in a proton transfer reaction

$$
A H+B \rightarrow A^{-}+H^{+} B
$$

it is reasonable to choose the DS function to be the difference between the bond being broken and that being formed,

$$
\xi\left(\mathbf{x}_{A^{-}}, \mathbf{x}_{B}, \mathbf{x}_{H^{+}}\right)=\left|\mathbf{x}_{H^{+}}-\mathbf{x}_{A^{-}}\right|-\left|\mathbf{x}_{H^{+}}-\mathbf{x}_{B}\right|
$$

On the other hand, for general complex reactions, it is not always possible to make a choice based solely on intuition. For example, if the above proton transfer reaction takes place in a polar solvent, the DS function defined in Eq. 1.3 may be too simple to describe the collective rearrangement of ambient solvent molecules induced by the proton transfer.

The work of Pollak ${ }^{14}$, for example, has shown in a simple model of a condensed phase reaction (a 1d reaction coordinate coupled to a harmonic bath), if the DS is chosen also to include the bath coordinates (as well as the $1 \mathrm{~d}$ reaction coordinate itself), then the frictional effects of Gtote-Hynes theory ${ }^{15}$ emerge from ordinary TST. The goal, therefore, is to have a general way of choosing a DS, one that is not system-specific and as 'automatic' as possible, and also simple enough for application of the QI theory to complex systems.

To this end we consider in this paper a family of planar DS's that are normal to the minimum energy path (MEP) at various points along it, connecting reactants and products. The MEP, also known as the Intrinsic Reaction Coordinate ${ }^{16}$, is the steepest descent path (in mass-weighted Cartesian coordinates) through the saddle point on a potential energy surface that connects reactants and products. Along the MEP all the atoms of the system are involved, and computing the MEP for a given system is now a common feature of modern quantum chemistry software packages such as Gaussian ${ }^{17}$. Though the MEP plays a central role in the Reaction Path Hamiltonian ${ }^{18}(\mathrm{RPH})$ description of chemical reactions, we emphasize that we are not using the RPH (with its curvilinear reaction coordinate, and local 
harmonic coordinates perpendicular to the MEP). The dynamical coordinates and momenta used to characterize the system throughout this paper are Cartesian; the MEP is used solely for the purpose of defining a family of DS's.

The paper is organized as follows: Section II first presents the basic idea and general methodology, first for a 'simple' Cartesian system (i.e., without rotation involved), and then for an $N$ atom system in 3d space. Section III briefly reviews the QI rate theory, and then Section IV presents the application of the approach to the simplest possible example, the collinear $\mathrm{H}+\mathrm{H}_{2}$ reaction. Section V concludes.

\section{GENERAL APPROACH}

\section{A. System without overall rotation and translation}

We first consider a generic Cartesian system of an arbitrary number of dimensions (degrees of freedom), for which the Hamiltonian is of standard form

$$
\begin{aligned}
\hat{H} & =\frac{\hat{\mathbf{p}}^{2}}{2}+V(\mathbf{x}), \\
\hat{\mathbf{p}} & =\frac{\hbar}{i} \frac{\partial}{\partial \mathbf{x}}
\end{aligned}
$$

where the Cartesian coordinates have been scaled so that all degrees of freedom have unit mass. Figure 1 shows the sketch of a contour plot for a $2 d$ potential energy surface with a saddle point; the dashed line denotes the MEP. The variable $s$ denotes the (arc length) distance along the MEP (with $s=0$ arbitrarily chosen at the saddle point), a $(s)$ are the Cartesian coordinates of the MEP at distance $s$ along it, and $\mathbf{n}(s) \equiv \mathbf{a}^{\prime}(s)$ is the normalized gradient vector (which points along the MEP),

$$
\mathbf{n}(s)=-\operatorname{sign}(s) \frac{\partial V}{\partial \mathbf{x}} /\left|\frac{\partial V}{\partial \mathbf{x}}\right|, \mathbf{x}=\mathbf{a}(s)
$$


where negative (positive) $s$ corresponds to the reactant (product) side of the saddle point. The solid line in Figure 1 is the planar (actually linear in this 2d picture) DS normal to the MEP at distance $s$ along it. The equation which defines this DS is of standard form

$$
\xi(\mathbf{x} ; s)=0
$$

where the function $\xi(\mathbf{x} ; s)$ is given by

$$
\xi(\mathbf{x} ; s)=\mathbf{n}(s) \cdot(\mathbf{x}-\mathbf{a}(s))
$$

i.e., the DS is defined by the criterion that the vector $\mathbf{x}-\mathbf{a}(s)$ is normal to the gradient vector $\mathbf{n}(s)$, which points along the MEP, at position $s$ along it; this in general defines a (hyper) plane normal to the DS at position $s$. We emphasize again that here $s$ is a parameter, not one of the coordinates of the system (which are the Cartesian coordinates $\mathbf{x}$ ). Eq (2.3) thus defines a 1-parameter family of DS's, normal to the MEP at distance $s$ along it. With a DS defined in general by Eq (2.3), the flux operator, which is needed in the QI rate expressions (and also in other rate theories), is given by

$$
\hat{F}=\frac{1}{2}\left[\hat{\mathbf{p}} \cdot \frac{\partial \xi(\mathbf{x})}{\partial \mathbf{x}} \delta(\xi(\mathbf{x}))+\delta(\xi(\mathbf{x})) \frac{\partial \xi(\mathbf{x})}{\partial \mathbf{x}} \cdot \hat{\mathbf{p}}\right] .
$$

Since the function $\xi(\mathbf{x} ; s)$ in Eq (2.3) for the present 1-parameter family of planar DS's is a linear function of $\mathbf{x}$, the flux operator takes on the following particularly simple form

$$
\hat{F}_{s}=\frac{1}{2}[\hat{\mathbf{p}} \cdot \mathbf{n}(s) \delta(\xi(\mathbf{x} ; s))+\delta(\xi(\mathbf{x} ; s)) \mathbf{n}(s) \cdot \hat{\mathbf{p}}]
$$

\section{B. N-atom system in $3 \mathrm{~d}$ space}

For a molecular system of $N$ atoms in $3 \mathrm{~d}$ space, we still wish to utilize the $3 N$ Cartesian coordinates for the atoms as the dynamical variables in which path integrals, for example, are evaluated. However, specifying the family of DS's as in Section IIA is more complicated 
because they should be invariant to overall rotation and translation of the total $N$ atom system.

The problem can be illustrated by considering a $2 \mathrm{~d}$ Cartesian system $\left(x_{1}=x, x_{2}=y\right)$ with rotational symmetry; i.e., the potential function $V$ depends only on the radial coordinate $r=\sqrt{x^{2}+y^{2}}$, and we assume that it has a barrier located at $r=r_{0}$ (see Fig 2a). In the $2 \mathrm{~d}$ space, there is thus a continuum of saddle points, indicated by the circle of radius $r_{0}$ in Fig 2b. The dashed line shows a MEP going through one particular saddle point; this is the MEP that would be generated by starting at the indicated saddle point and following the gradient vector downward to the inside of the circle ('reactants') and also outside ('products'). The dotted line in Fig $2 \mathrm{~b}$ is the DS that is normal (actually linear in this $2 \mathrm{~d}$ picture) to this MEP at position $s$ along it, as specified by the criterion described in Section IIA, and one can now see the shortcoming of using this planar DS: the point $\mathbf{x}$ in the $2 \mathrm{~d}$ space shown in Fig $2 \mathrm{~b}$ is on the 'reactant' side of the planar DS, whereas it is in fact on the 'product' side of the true DS (the circle).

To remedy the situation one needs to rotate the position coordinate $\mathbf{x}$ by an angle $\theta$,

$$
\mathbf{x}^{\prime}=\mathbf{R}(\theta) \cdot \mathbf{x}
$$

where

$$
\mathbf{R}(\theta)=\left(\begin{array}{cc}
\cos (\theta) & \sin (\theta) \\
-\sin (\theta) & \cos (\theta)
\end{array}\right)
$$

choosing $\theta$ so that $\mathbf{x}^{\prime}$ is as close as possible to the position a on the MEP. It is clear from Fig. $2 \mathrm{~b}$ that this rotated position $\mathbf{x}^{\prime}$ will lie along the same radial direction as the point $\mathbf{a}$. One can now apply the criterion of Section IIA to the rotated position $\mathbf{x}^{\prime}$ to obtain the function which defines the DS,

$$
\xi(\mathbf{x})=\mathbf{n} \cdot(\mathbf{R}(\theta) \cdot \mathbf{x}-\mathbf{a})
$$


It should be clear that this procedure produces a DS that is equivalent to the circle in Fig $2 \mathrm{~b}$, while still utilizing the Cartesian coordinates $\mathbf{x}=\left(x_{1}, x_{2}\right)$ as the dynamical variables of the system. It is now clear how to generalize this idea to the case of $N$ atoms in $3 \mathrm{~d}$ space. (One must also take account of overall translational invariance, but this is easy.) Let $x_{i}, i=1,2,3$ denote the three mass-weighted $\left(x_{i}=\sqrt{m_{i}} r_{i}\right)$ Cartesian coordinates of atom $i$, and $\mathbf{x}=\left(\mathbf{x}_{1}, \mathbf{x}_{2}, \cdots, \mathbf{x}_{N}\right)$ the $3 N$ Cartesian coordinates of the entire system, and let $\mathbf{a}(s)$ similarly denote the $3 N$ Cartesian coordinates along a MEP as a function of $s$, the (signed) distance along it. Analogous to the $2 \mathrm{~d}$ plane rotation matrix of $\mathrm{Eq}(2.6 \mathrm{~b})$, we introduce the $3 \times 3$ rotation matrix $\mathbf{R}(\phi, \theta, \chi)$, parameterized by three Euler angles, which rotates a 3 -vector in $3 \mathrm{~d}$ space. The idea is to rotate each vector $\mathbf{x}_{i}$, i.e., the entire $N$ atom system, so that the $3 N$ vector $\mathbf{x}$ is as close as possible to the point $\mathbf{a}(s)$ on the MEP at distance $s$ along it.

One must carry out the rotation of the atomic vectors about the center of mass of the $N$ atom system. Thus if $\mathbf{X}_{0}$ and $\mathbf{A}_{0}$ are the mass-weighted centers of mass of point $\mathbf{x}$ and point $\mathbf{a}(s)$, respectively -

$$
\begin{aligned}
\mathbf{X}_{0} & =\sum_{i=1}^{N} \sqrt{\frac{m_{i}}{M}} \mathbf{x}_{i} \\
\mathbf{A}_{0}(s) & =\sum_{i=1}^{N} \sqrt{\frac{m_{i}}{M}} \mathbf{a}_{i}(s),
\end{aligned}
$$

where $M$ is the total mass,

$$
M=\sum_{i=1}^{N} m_{i}
$$

then the function $\xi(\mathbf{x} ; s)$ that defines the (hyper) planar DS at distance $s$ along the MEP is

$$
\xi(\mathbf{x} ; s)=\sum_{i=1}^{N} \mathbf{n}_{i}(s) \cdot\left\{\mathbf{R}(\phi, \theta, \chi) \cdot\left(\mathbf{x}_{i}-\mathbf{X}_{0}\right)-\left[\mathbf{a}_{i}(s)-\mathbf{A}_{0}(s)\right]\right\},
$$

where for each point $\mathbf{x}$ (and each position $\mathbf{a}(s)$ along the MEP) one chooses the Euler angles $(\phi, \theta, \chi)$ to minimize the distance between $\mathbf{x}$ and $\mathbf{a}(s)$. I.e., before projecting the difference 
of $\mathbf{x}$ and $\mathbf{a}(s)$ onto the gradient vector $\mathbf{n}(s)$ pointing along the MEP, as in Section IIA, we rotate $\mathbf{x}$ (about its center of mass) to make it as close as possible to $\mathbf{a}(s)$ (relative to its center of mass). This rotation step is an awkward aspect of the procedure, but since it involves no evaluations of the potential energy surface it should cause minimal additional computational effort.

If one were to generate the MEP in internal coordinates, ${ }^{19,20}$ then one could avoid this step of having to rotate the configuration point $\mathbf{x}$ to 'line up' (i.e., be as close as possible to) the point $\mathbf{a}(s)$ on the MEP. Use of internal coordinates, however, has its own difficulties. Also, in a very large molecular system (e.g., a chemical reaction in solution), it is unlikely that overall rotation of the entire system will be of concern. Furthermore, one will most likely choose the planar DS only to involve the coordinates of the those atoms involved in the reaction (and perhaps a first solvation shell), again meaning that overall rotation of the system will not be involved.

\section{APPLICATION TO THE QI MODEL}

\section{A. The QI approximation of the canonical rate constant of chemical reaction}

To see how to use the above DS's within the QI rate theory, we first give a brief review of the working expressions of the QI model (details can be found in Ref. 4). For a general chemical reaction that involves $N$ atoms in three-dimensional space, the QI approximation gives the thermal rate constant as

$$
k_{Q I}(T)=\frac{1}{Q_{r}} C_{f f}(0) \frac{\sqrt{\pi}}{2} \frac{\hbar}{\Delta H},
$$

where $Q_{r}$ is the reactant partition function per unit volume, $C_{f f}(0)$ is the zero time value of the flux-flux correlation function with two separate dividing surfaces,

$$
C_{f f}(t)=\operatorname{tr}\left[e^{-\beta \hat{H} / 2} \hat{F}_{s_{1}} e^{-\beta \hat{H} / 2} e^{i \hat{H} t / \hbar} \hat{F}_{s_{2}} e^{-\hat{H} t / \hbar}\right]
$$


where $\beta$ is the inverse temperature $1 /\left(k_{B} T\right), \hat{H}$ is the Hamiltonian given in Eq. (2.3), and $\hat{F}_{s_{1}}$ and $\hat{F}_{s_{2}}$ are the flux operators defined by Eq (2.4) with $s_{1}$ and $s_{2}$ indicating two different dividing surfaces. $\Delta H$ in Eq. (3.1) is a specific type of energy variance, given by

$\Delta H^{2}=\frac{\operatorname{tr}\left[\Delta\left(\xi\left(\hat{\mathbf{x}} ; s_{1}\right)\right) e^{-\beta \hat{H} / 2} \hat{H}^{2} \Delta\left(\xi\left(\hat{\mathbf{x}} ; s_{2}\right)\right) e^{-\beta \hat{H} / 2}-\Delta\left(\xi\left(\hat{\mathbf{x}} ; s_{1}\right)\right) e^{-\beta \hat{H} / 2} \hat{H} \Delta\left(\xi\left(\hat{\mathbf{x}} ; s_{2}\right)\right) e^{-\beta \hat{H} / 2} \hat{H}\right]}{\operatorname{tr}\left[\Delta\left(\xi\left(\hat{\mathbf{x}} ; s_{1}\right)\right) e^{-\beta \hat{H} / 2} \Delta\left(\xi\left(\hat{\mathbf{x}} ; s_{2}\right)\right) e^{-\beta \hat{H} / 2}\right]}$

with $\Delta(\xi(\hat{\mathbf{x}} ; s))$ being a modified version of the Dirac delta function

$$
\Delta(\xi(\hat{\mathbf{x}} ; s))=\delta(\xi(\hat{\mathbf{x}} ; s)) \sqrt{\sum_{i=1}^{N}\left|\nabla_{i} \xi(\hat{\mathbf{x}} ; s)\right|^{2}} .
$$

\section{B. Path integral representation}

To be convenient for path integral evaluation, the rate constant expression Eq. (3.1) is rewritten as ${ }^{10}$

$$
k_{Q I}(T)=\frac{C_{d d}(0)}{Q_{r}} \frac{C_{f f}(0)}{C_{d d}(0)} \frac{\sqrt{\pi}}{2} \frac{\hbar}{\Delta H}
$$

where $C_{d d}(0)$ is the zero time value of the delta-delta correlation function

$$
C_{d d}(t)=\operatorname{tr}\left[\Delta\left(\xi\left(\hat{\mathbf{x}} ; s_{1}\right)\right) e^{-\beta \hat{H} / 2} e^{i \hat{H} t / \hbar} \Delta\left(\xi\left(\hat{\mathbf{x}} ; s_{2}\right)\right) e^{-\beta \hat{H} / 2} e^{-i \hat{H} t / \hbar}\right] .
$$

The path integral expression for $C_{d d}(0)$ is

$$
\begin{aligned}
C_{d d}(0)= & \prod_{i=1}^{N}\left(\frac{P}{2 \pi \hbar^{2} \beta}\right)^{3 P / 2} \int d \mathbf{x}^{(1)} \int d \mathbf{x}^{(2)} \cdots \int d \mathbf{x}^{(P)} \Delta\left(\xi\left(\mathbf{x}^{(0)} ; s_{1}\right)\right) \Delta\left(\xi\left(\mathbf{x}^{(P / 2)} ; s_{2}\right)\right) \\
& \times \exp \left[-\beta \Phi\left(\mathbf{x}^{(1)}, \mathbf{x}^{(2)}, \cdots, \mathbf{x}^{(P)}\right)\right],
\end{aligned}
$$

where $P$ is the number of imaginary time slices, $\mathbf{x}^{(k)}=\left(\mathbf{x}_{1}^{(k)}, \mathbf{x}_{2}^{(k)}, \cdots, \mathbf{x}_{N}^{(k)}\right)$ denotes the $3 \mathrm{~N}$-dimensional Cartesian coordinates of the system for the $k$ th time slice, and $\Phi\left(\mathbf{x}^{(1)}, \mathbf{x}^{(2)}, \cdots, \mathbf{x}^{(P)}\right)$ is the discretized action given by

$$
\begin{aligned}
\Phi\left(\mathbf{x}^{(1)}, \mathbf{x}^{(2)}, \cdots, \mathbf{x}^{(P)}\right)= & \frac{P}{2 \hbar^{2} \beta^{2}} \sum_{k=1}^{P} \sum_{i=1}^{N} m_{i}\left(\mathbf{x}_{i}^{(k)}-\mathbf{x}_{i}^{(k-1)}\right)^{2} \\
& +\frac{1}{P} \sum_{k=1}^{P} V\left(\mathbf{x}_{1}^{(k)}, \mathbf{x}_{2}^{(k)}, \cdots, \mathbf{x}_{N}^{(k)}\right)
\end{aligned}
$$


with $\mathbf{x}^{(0)}=\mathbf{x}^{(P)}$. The path integral expression for the flux-flux correlation function is then given by

$$
\begin{aligned}
C_{f f}(0)= & \prod_{i=1}^{N}\left(\frac{P}{2 \pi \hbar^{2} \beta}\right)^{3 P / 2} \int d \mathbf{x}^{(1)} \int d \mathbf{x}^{(2)} \cdots \int d \mathbf{x}^{(P)} f_{v}\left(\mathbf{x}^{(1)}, \mathbf{x}^{(2)}, \cdots, \mathbf{x}^{(P)}\right) \\
& \times \Delta\left(\xi\left(\mathbf{x}^{(0)} ; s_{1}\right)\right) \Delta\left(\xi\left(\mathbf{x}^{(P / 2)} ; s_{2}\right)\right) \exp \left[-\beta \Phi\left(\mathbf{x}^{(1)}, \mathbf{x}^{(2)}, \cdots, \mathbf{x}^{(P)}\right)\right]
\end{aligned}
$$

i.e., it is the same as $C_{d d}(0)$ with the following additional factor in the integrand,

$$
\begin{aligned}
f_{v}\left(\mathbf{x}^{(1)}, \mathbf{x}^{(2)}, \cdots, \mathbf{x}^{(P)}\right)= & \left(\frac{i P}{2 \hbar \beta}\right)^{2} \frac{\sum_{i=1}^{N} \nabla_{i} \xi\left(\mathbf{x}^{(0)} ; s_{1}\right) \cdot\left(\mathbf{x}_{i}^{(1)}-\mathbf{x}_{i}^{(P-1)}\right)}{\sqrt{\sum_{i=1}^{N}\left|\nabla_{i} \xi\left(\mathbf{x}^{(0)} ; s_{1}\right)\right|^{2}}} \\
& \times \frac{\sum_{i=1}^{N} \nabla_{i} \xi\left(\mathbf{x}^{\left(\frac{P}{2}\right)} ; s_{2}\right) \cdot\left(\mathbf{x}_{i}^{\left(\frac{P}{2}+1\right)}-\mathbf{x}_{i}^{\left(\frac{P}{2}-1\right)}\right)}{\sqrt{\sum_{i=1}^{N}\left|\nabla_{i} \xi\left(\mathbf{x}^{\left(\frac{P}{2}\right)} ; s_{2}\right)\right|^{2}}}
\end{aligned}
$$

The energy variance is given by

$$
\Delta H=\hbar\left[-\frac{1}{2} \frac{\ddot{C}_{d d}(0)}{C_{d d}(0)}\right]
$$

with

$$
\begin{aligned}
\ddot{C}_{d d}(0)= & =-\frac{1}{\hbar^{2}} \prod_{i=1}^{N}\left(\frac{P}{2 \pi \hbar^{2} \beta}\right)^{3 P / 2} \int d \mathbf{x}^{(1)} \int d \mathbf{x}^{(2)} \cdots \int d \mathbf{x}^{(P)} \\
& \times\left[F^{2}\left(\mathbf{x}^{(1)}, \mathbf{x}^{(2)}, \cdots, \mathbf{x}^{(P)}\right)+G\left(\mathbf{x}^{(1)}, \mathbf{x}^{(2)}, \cdots, \mathbf{x}^{(P)}\right)\right] \\
& \times \Delta\left(\xi\left(\mathbf{x}^{(0)} ; s_{1}\right)\right) \Delta\left(\xi\left(\mathbf{x}^{(P / 2)} ; s_{2}\right)\right) \exp \left[-\beta \Phi\left(\mathbf{x}^{(1)}, \mathbf{x}^{(2)}, \cdots, \mathbf{x}^{(P)}\right)\right],
\end{aligned}
$$

where

$$
\begin{aligned}
F\left(\mathbf{x}^{(1)}, \mathbf{x}^{(2)}, \cdots, \mathbf{x}^{(P)}\right)= & \frac{P}{\hbar^{2} \beta^{2}}\left\{\sum_{k=1}^{P / 2}-\sum_{k=P / 2+1}^{P}\right\} \sum_{i=1}^{N}\left(\mathbf{x}_{i}^{(k)}-\mathbf{x}_{i}^{(k-1)}\right)^{2} \\
& +\frac{2}{P}\left\{\sum_{k=1}^{P / 2-1}-\sum_{k=P / 2+1}^{P-1}\right\} V\left(\mathbf{x}_{i}^{(k)}, \mathbf{x}_{2}^{(k)}, \cdots, \mathbf{x}_{N}^{(k)}\right)
\end{aligned}
$$

and

$$
\left.G\left(\mathbf{x}^{(1)}, \mathbf{x}^{(2)}, \cdots, \mathbf{x}^{(P)}\right)=\frac{6 N P}{\beta^{2}}-\frac{4 P}{\hbar^{2} \beta^{3}} \sum_{k=P / 2+1}^{P}\right\} \sum_{i=1}^{N}\left(\mathbf{x}_{i}^{(k)}-\mathbf{x}_{i}^{(k-1)}\right)^{2}
$$


If the Monte Carlo weight function is chosen as

$$
\begin{aligned}
\rho\left(\mathbf{x}^{(1)}, \mathbf{x}^{(2)}, \cdots, \mathbf{x}^{(P)}\right)= & \prod_{i=1}^{N}\left(\frac{m_{i} P}{2 \pi \hbar^{2} \beta}\right)^{3 P / 2} \Delta\left(\xi ( \mathbf { x } ^ { ( 0 ) } ; s _ { 1 } ) \Delta \left(\xi\left(\mathbf{x}^{(P / 2)} ; s_{2}\right)\right.\right. \\
& \times \exp \left[-\beta \Phi\left(\mathbf{x}^{(1)}, \mathbf{x}^{(2)}, \cdots, \mathbf{x}^{(P)}\right)\right],
\end{aligned}
$$

and the ensemble average thus defined as

$$
\langle\cdots\rangle=\frac{\int d \mathbf{x}^{(1)} \int d \mathbf{x}^{(2)} \cdots \int d \mathbf{x}^{(P)} \rho\left(\mathbf{x}^{(1)}, \mathbf{x}^{(2)}, \cdots, \mathbf{x}^{(P)}\right)(\cdots)}{\int d \mathbf{x}^{(1)} \int d \mathbf{x}^{(2)} \cdots \int d \mathbf{x}^{(P)} \rho\left(\mathbf{x}^{(1)}, \mathbf{x}^{(2)}, \cdots, \mathbf{x}^{(P)}\right)} ;
$$

then the ratio $C_{f f}(0) / C_{d d}(0)$ and $\Delta H$ can be obtained by one Monte Carlo simulation,

$$
\frac{C_{f f}(0)}{C_{d d}(0)}=\left\langle f_{v}\left(\mathbf{x}^{(1)}, \mathbf{x}^{(2)}, \cdots, \mathbf{x}^{(P)}\right)\right\rangle
$$

and

$$
\Delta H^{2}=\frac{1}{2}\left\langle F^{2}\left(\mathbf{x}^{(1)}, \mathbf{x}^{(2)}, \cdots, \mathbf{x}^{(P)}\right)+G\left(\mathbf{x}^{(1)}, \mathbf{x}^{(2)}, \cdots, \mathbf{x}^{(P)}\right)\right\rangle
$$

respectively.

The delta function in the right side of Eq. (3.4) is approximated by a Gaussian with sufficiently small width, so that

$$
\Delta(\xi(\mathbf{x} ; s)) \approx \frac{1}{\sqrt{2 \pi} \sigma} \exp \left[-\frac{1}{2}\left(\frac{\xi(\mathbf{x} ; s)}{\sigma}\right)^{2}\right] \sqrt{\sum_{i=1}^{N}\left|\nabla_{i} \xi(\hat{\mathbf{x}} ; s)\right|^{2}} .
$$

As pointed out in Ref. 15 , the ratio $C_{f f}(0) / C_{d d}(0)$ and $\Delta H$ can be evaluated straightforwardly by the constrained equilibrium simulation. While, the computation of $C_{d d}(0) / Q_{r}$ involves in some tricks to handle the statistical sampling of rare events ${ }^{21,22}$, because $C_{d d}(0)$ is a property associated with the top of the potential barrier while $Q_{r}$ is that with the bottom of the reactant well. In our applications, we rewrite $C_{d d}\left(0 ; s_{1}, s_{2}\right) / Q_{r}$ as follows:

$$
\frac{C_{d d}\left(0 ; s_{1}, s_{2}\right)}{Q_{r}}=\frac{C_{d d}\left(0 ; s_{1}, s_{2}\right)}{C_{d d}\left(0 ; s_{r}, s_{r}\right)} \frac{C_{d d}\left(0 ; s_{r}, s_{r}\right)}{Q_{r}},
$$

here $s_{r}$ is chosen so that the dividing surface is deep in the reactant region. The first ratio on the right side of above equation is calculated via umbrella sampling technique (see Appendix A). The second ratio can be evaluated analytically. 


\section{NUMERICAL TEST}

Now we present the numerical results of a test for the collinear $H+H_{2}$ reaction. The QI rate calculations were carried out at temperature $300 K$ on the TK potential energy surface $^{23}$. We used one dividing surface in our calculations, since earlier work ${ }^{7,10}$ showed that this system has only one saddle point on the quantum free surface at $300 K$.

In normal mode coordinates about the saddle point, the Hamiltonian for the collinear system is

$$
\hat{H}=-\frac{1}{2 \mu}\left(\frac{\partial^{2}}{\partial x^{2}}+\frac{\partial^{2}}{\partial y^{2}}\right)+V(x, y),
$$

where the reduced mass $\mu=1224.0 \mathrm{au}$. Figure 3 shows a contour plot of the PES.

Since this $2 \mathrm{~d}$ collinear system does not involve overall rotation or translation, one can use the dividing surface defined in Eq. (2.3). 45 points are evenly distributed along the MEP shown in Fig 3. The location of each point is indicated by the parameter $s$, the distance along the MEP. The ratio $C_{f f}(0 ; s) / C_{d d}(0 ; s)$ and $\Delta H$ are calculated for each point with $s \in[-0.62,0]$. A sequence of umbrella samplings are carried out along the MEP to obtain the ratio $C_{d d}(0 ; s) / C_{d d}\left(0 ; s_{r}\right)$ with $s_{r}$ denoting the value of $s$ of the end point in the reactant region (see Appendix $A$ for details). The ratio $C_{d d}\left(0 ; s_{r}\right) / Q_{r}$ can be evaluated analytically as

$$
\frac{C_{d d}\left(0 ; s_{r}\right)}{Q_{r}}=\sqrt{\frac{2 \mu}{\pi \beta}}
$$

In path integral calculations, the number of imaginary time slices is 60 , and the number of Monte Carlo cycles is $8 \times 10^{6}$, which is sufficient to converge the relevant quantities within $2 \%$ statistical error. One practical difficulty we encounter is that some sampling points leak into the product region because the potential barrier along the dividing surface is not high enough to separate the reactant and product region. In order to confine all sampling points in the reactant region, we constrain the bond length of the hydrogen molecule $r$ to be less than two times of its equilibrium length $r_{e}$.

Figure 4 shows the quantum free energy, $F=-\log \left[C_{d d}(0 ; s) / C_{d d}\left(0 ; s_{r}\right)\right] / \beta$, in the vicinity 
of the saddle point. The DVR result is also provided in Figure 4 to make sure that the umbrella sampling gives accurate results for $C_{d d}(0 ; s) / C_{d d}\left(0 ; s_{r}\right)$ along the MEP. The ratio $C_{f f}(0 ; s) / C_{d d}(0 ; s)$ and $\Delta H(s)$ are shown in Fig 5 and Fig 6 respectively. These two quantities vary relatively slowly as the dividing surface moving around the top of barrier. Figure 7 shows that the QI rate is quit flat when the dividing surface is close to top of barrier and then increases dramatically as the dividing surface moving further away from the top of barrier.

\section{CONCLUDING REMARKS}

In this paper, we have presented a simple but general way of choosing a family of DS's for application of the quantum instanton approximation for thermal rate constants. In our definition, the family of planar DS's is normal to the MEP at various points along it so that it can correctly describe the collective motion of all atoms invovled in the reaction. The location of the DS along the MEP is controlled by one parameter, $s$, the distance along the MEP, so that the QI variational criterion for choosing DS's is reduced to a two-variable optimization problem which is more tractable for general system with many degrees of freedom. We emphasize again, that the "reaction coordinate" $s$ is not one of the dynamical coordinates of the system (which we assume will be the Cartesian coordinates); it is simply a parameter that specifies the location of the planar DS.

To illustrate this approach, we have applied it to the PIMC evaluation of the QI rate of the collinear $\mathrm{H}+\mathrm{H}_{2}$ reaction. The results of PIMC evaluation are in excellent agreement with those of a standard quantum (e.g., DVR) calculation. Comparing with the previous PIMC implementation of the QI theory, a major modification here is made in the umbrella sampling procedure for the quantum free energy calculation, which is necessary because each DS defined in Eq (2.3) is locally associated with one point on the MEP. 


\section{Acknowledgments}

This work was supported in part by the office of Naval Research Grant Number N0001401-1-0236, and by Office of Science, Office of Basic Energy Sciences, Chemical Sciences, Geosciences, and Biosciences Division, U.S. Department of Energy under Contract No. DEAC02-05CH11231. We also acknowledge a generous allocation of supercomputing time from the National Energy Research Scientific Computing Center (NERSC). We would like to thank Dr. Michele Ceotto for many useful discussions.

\section{Appendix A: EVALUATING $C_{d d}(0 ; s) / C_{d d}\left(0 ; s_{r}\right)$ VIA THE UMBRELLA SAMPLING TECHNIQUE}

First we note that, once we know the probability density

$$
P(s)=\frac{\int d \mathbf{x}^{(0)} \int d \mathbf{x}^{(1)} \cdots \int d \mathbf{x}^{(P)} \delta\left[\xi\left(\mathbf{x}^{(0)} ; s\right)\right] \delta\left[\xi\left(\mathbf{x}^{(P / 2)} ; s\right)\right] e^{-\beta \Phi^{\prime}\left(\mathbf{x}^{(1)}, \mathbf{x}^{(2)}, \cdots, \mathbf{x}^{(P)}\right)}}{\int d \mathbf{x}^{(0)} \int d \mathbf{x}^{(1)} \cdots \int d \mathbf{x}^{(P)} e^{-\beta \Phi^{\prime}\left(\mathbf{x}^{(1)}, \mathbf{x}^{(2)}, \cdots, \mathbf{x}^{(P)}\right)}}
$$

with

$$
\begin{aligned}
\Phi^{\prime}\left(\mathbf{x}^{(1)}, \mathbf{x}^{(2)}, \cdots, \mathbf{x}^{(P)}\right)= & \Phi\left(\mathbf{x}^{(1)}, \mathbf{x}^{(2)}, \cdots, \mathbf{x}^{(P)}\right)-\frac{1}{2 \beta} \log \left(\sum_{i=1}^{N}\left|\nabla_{i} \xi\left(\mathbf{x}^{(0)} ; s\right)\right|^{2}\right) \\
& -\frac{1}{2 \beta} \log \left(\sum_{i=1}^{N}\left|\nabla_{i} \xi\left(\mathbf{x}^{(P / 2)} ; s\right)\right|^{2}\right),
\end{aligned}
$$

then

$$
\frac{C_{d d}(0 ; s)}{C_{d d}\left(0 ; s_{r}\right)}=\frac{P(s)}{P\left(s_{r}\right)}
$$

However, due to the exponentially small probability density in the region around the top of barrier, we use the umbrella sampling technique to evaluate $P(s)$ along the MEP. Using a set of umbrella potentials $\left\{U_{m}(\mathbf{x}), m=1,2, \cdots, M\right\}$ which forces Monte Carlo procedure to sample a sequence of sub-configurations along the MEP, we can obtain a set of probability density distributions, $\left\{P_{m}(s), m=1,2, \cdots, M\right\}$ in these sub-configurations, and 


$$
P_{m}(s)=\frac{\int d \mathbf{x}^{(0)} \int d \mathbf{x}^{(1)} \cdots \int d \mathbf{x}^{(P)} \delta\left[\xi\left(\mathbf{x}^{(0)} ; s\right)\right] \delta\left[\xi\left(\mathbf{x}^{(P / 2)} ; s\right)\right] e^{-\beta \Phi_{m}\left(\mathbf{x}^{(1)}, \mathbf{x}^{(2)}, \cdots, \mathbf{x}^{(P)}\right)}}{\int d \mathbf{x}^{(0)} \int d \mathbf{x}^{(1)} \cdots \int d \mathbf{x}^{(P)} e^{-\beta \Phi_{m}\left(\mathbf{x}^{(1)}, \mathbf{x}^{(2)}, \cdots, \mathbf{x}^{(P)}\right)}}
$$

with

$$
\Phi_{m}\left(\mathbf{x}^{(1)}, \mathbf{x}^{(2)}, \cdots, \mathbf{x}^{(P)}\right)=\Phi^{\prime}\left(\mathbf{x}^{(1)}, \mathbf{x}^{(2)}, \cdots, \mathbf{x}^{(P)}\right)+U_{m}\left(\mathbf{x}^{(0)}\right)+U_{m}\left(\mathbf{x}^{(P / 2)}\right) .
$$

Then we find out $P(s)$ by requiring that $A_{m}(s)=-\log P_{m}(s) / \beta$, is a continuous function from one sub-configuration to the next. Usually, the umbrella potential is a function of the reaction coordinate $\xi$, but the reaction coordinate in our definition is locally associated with a point $\mathbf{a}(s)$ on the MEP, so it is difficult to set up a umbrella potential with respect to this reaction coordinate. In our applications to atom-diatom reaction $A+B C$, the umbrella potential is a function of distances $\left(r_{A B}, r_{B C}, r_{C A}\right)$,

$$
U_{m}(\mathbf{x})=\left\{\begin{array}{cc}
0 & \min \left(r_{A B}, r_{C A}\right) \in\left[b_{m-1}, b_{m+1}\right] \\
\infty & \text { otherwise }
\end{array}\right.
$$

for sampling in the reactant region. In this simulation, 7 umbrella potentials are used in the reactant side with $\left(b_{0}, b_{1}, \cdots, b_{8}\right)=(1.0,1.5,2.0,2.5,3.0,4.0,6.0,8.0,9.0)$ in a.u..

In the numerical simulations, the probability density distributions $P_{m}(s)$ are actually associated with a set of points $\left\{\mathbf{a}\left(s_{i}\right), i=1,2, \cdots, M\right\}$ on the MEP and the delta function is replaced by a Gaussian with sufficiently small width, so the final working expression is

$$
P_{m}\left(s_{i}\right)=\frac{1}{2 \pi \sigma^{2}}\left\langle\exp \left\{-\frac{1}{2 \sigma^{2}}\left[\xi^{2}\left(\mathbf{x}^{(0)} ; s_{i}\right)+\xi^{2}\left(\mathbf{x}^{(P / 2)} ; s_{i}\right)\right]\right\}\right\rangle_{m},
$$

where $\langle\cdots\rangle_{m}$ denotes the ensemble average

$$
\langle\cdots\rangle_{m}=\frac{\int d \mathbf{x}^{(0)} \int d \mathbf{x}^{(1)} \cdots \int d \mathbf{x}^{(P)} e^{-\beta \Phi_{m}\left(\mathbf{x}^{(1)}, \mathbf{x}^{(2)}, \cdots, \mathbf{x}^{(P)}\right)}(\cdots)}{\int d \mathbf{x}^{(0)} \int d \mathbf{x}^{(1)} \cdots \int d \mathbf{x}^{(P)} e^{-\beta \Phi_{m}\left(\mathbf{x}^{(1)}, \mathbf{x}^{(2)}, \cdots, \mathbf{x}^{(P)}\right)}} .
$$


1 The developement of transition-state theory is associated with the names of Wigner, Pelzer, Polanyi, Evans, and particularly Eyring; see, for example, the brief historical discussion and bibliography in K. J. Laidler, "Theories of Chemical Kinetics", McGraw-Hill, New York, N.Y., 1969, pp 41-43. The classic textbook refernce to traditional transition-state theory is S. Gladstone, K. J. Laidler, and H. Eyring, "Theory of Rate Processes", McGraw-Hill, New York, N.Y. 1941.

2 E. Wigner, J. Chem. Phys., 5, 720 (1937).

3 P. Pechukas and F. J. McLafferty, J. Chem. Phys., 58, 1622 (1973).

4 F. J. McLafferty and P. Pechukas, Chem. Phys. Lett., 27, 511 (1974).

5 W. H. Miller, Acct. Chem. Res., 9, 306 (1976).

6 J. W. Tromp and W. H. Miller, Faraday Disc. Chem. Soc., 84, 441 (1987).

7 W. H. Miller, Y. Zhao, M. Ceotto, and S. Yang, J. Chem. Phys., 119, 1329 (2003).

8 C. Venkataraman and W. H. Miller, J. Phys. Chem. A, 108, 3035 (2004).

9 W. H. Miller, J. Chem. Phys., 62, 1899 (1975).

10 T. Yamamoto and W. H. Miller, J. Chem. Phys., 120, 3086 (2004).

11 Y. Zhao, T. Yamamoto, and W. H. Miller, J. Chem. Phys., 120, 3100 (2004).

12 T. Yamamoto and W. H. Miller, J. Chem. Phys., 122, 044106 (2005).

13 J. Vanicek, W. H. Miller, J. F. Castillo, and F. J. Aoiz, J. Chem. Phys., 123, 054108 (2005).

14 E. Pollak, J. Chem. Phys., 85, 865 (1986).

15 R. F. Grote and J. T. Hynes, J. Chem. Phys., 73, 2715 (1980).

16 K. Fukui, Acct. Chem. Res., 14, 363 (1981).

17 M. J. Frisch, G. W. Trucks, H. B. Schlegel, G. E. Scuseria, M. A. Robb, J. R. Cheeseman, J. A. Montgomery, Jr., T. Vreven, K. N. Kudin, J. C. Burant, J. M. Millam, S. S. Iyengar, J. Tomasi, V. Barone, B. Mennucci, M. Cossi, G. Scalmani, N. Rega, G. A. Petersson, H. Nakatsuji, M. Hada, M. Ehara, K. Toyota, R. Fukuda, J. Hasegawa, M. Ishida, T. Nakajima, Y. Honda, 
O. Kitao, H. Nakai, M. Klene, X. Li, J. E. Knox, H. P. Hratchian, J. B. Cross, V. Bakken, C. Adamo, J. Jaramillo, R. Gomperts, R. E. Stratmann, O. Yazyev, A. J. Austin, R. Cammi, C. Pomelli, J. W. Ochterski, P. Y. Ayala, K. Morokuma, G. A. Voth, P. Salvador, J. J. Dannenberg, V. G. Zakrzewski, S. Dapprich, A. D. Daniels, M. C. Strain, O. Farkas, D. K. Malick, A. D. Rabuck, K. Raghavachari, J. B. Foresman, J. V. Ortiz, Q. Cui, A. G. Baboul, S. Clifford, J. Cioslowski, B. B. Stefanov, G. Liu, A. Liashenko, P. Piskorz, I. Komaromi, R. L. Martin, D. J. Fox, T. Keith, M. A. Al-Laham, C. Y. Peng, A. Nanayakkara, M. Challacombe, P. M. W. Gill, B. Johnson, W. Chen, M. W. Wong, C. Gonzalez, and J. A. Pople, Gaussian 03, Revision C.02. 18 W. H. Miller, N. C. Handy, and J. E. Adams, J. Chem. Phys., 72, 99 (1980).

19 C. F. Jackels, Z. Gu, and D. G. Truhlar, J. Chem. Phys., 102, 3188 (1995).

20 K. A. Nguyen, C. F. Jackels, and D. G. Truhlar, J. Chem. Phys., 104, 6491 (1996).

21 D. Chandler, J. Chem. Phys., 68, 2959 (1978).

22 D. Chandler, Introduction to Modern Statistical Mechanics, Oxford Univ. Press, Oxford, 1987.

23 D. G. Truhlar and A. Kuppermann, J. Chem. Phys., 56, 2232 (1972). 


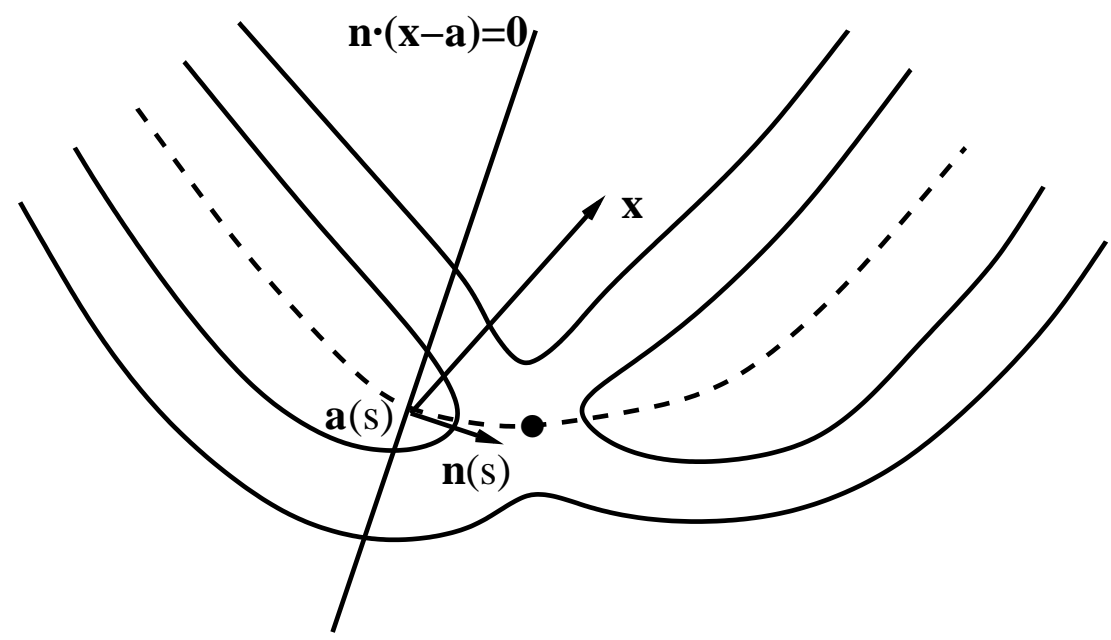

Figure 1: A contour of potential energy surface. The dashed line indicates a MEP from the reactant to the product region. $\mathbf{a}(s)$ is a point on the MEP, and $\mathbf{n}(s)$ is the tangent vector of the MEP. $s$ is the arc length from a to the saddle point (indicated by a big black dot) along the MEP. The dividing surface determined by Eq. (2.3) is a line normal to the MEP at the point $\mathbf{a}(s)$. 


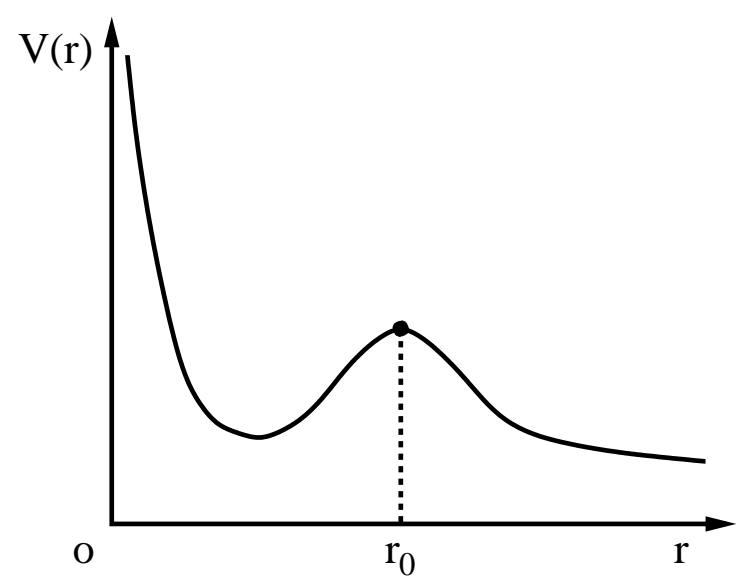

(a)

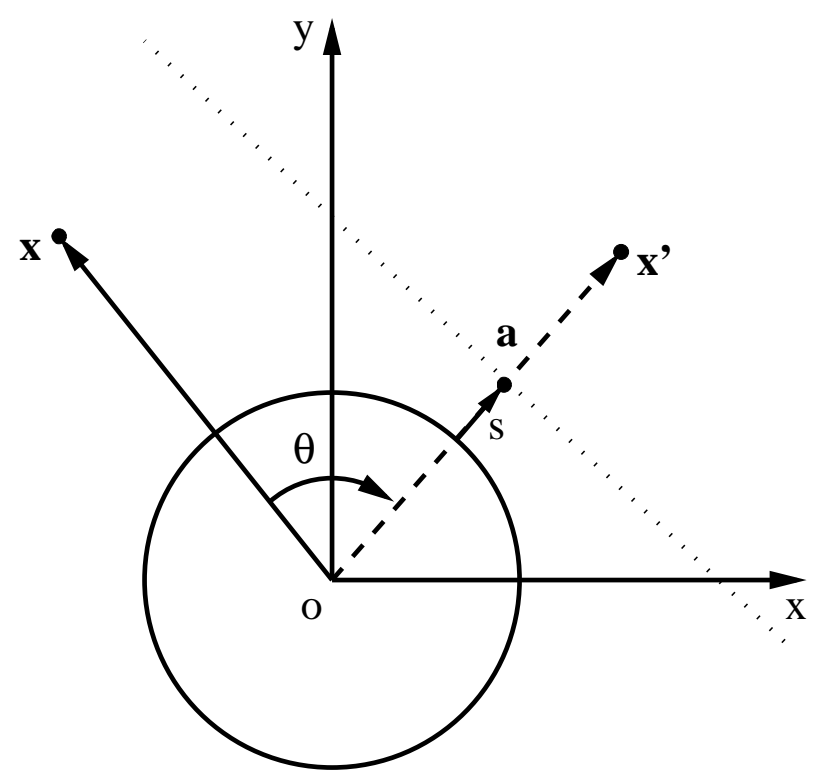

(b)

Figure 2: (a) PES as a function of the radial coordinate $r$. The top of barrier is located at $r=r_{0}$. (b) The DS in 2d Cartesian coordinates. The dashed line is a MEP passing through one particular saddle point. a is a point at position $s$ along the MEP. The DS at top of barrier is a circle with the radius equal to $r_{0}$. The dotted line indicates the planar DS defined by Eq. (2.3a) at point a. 


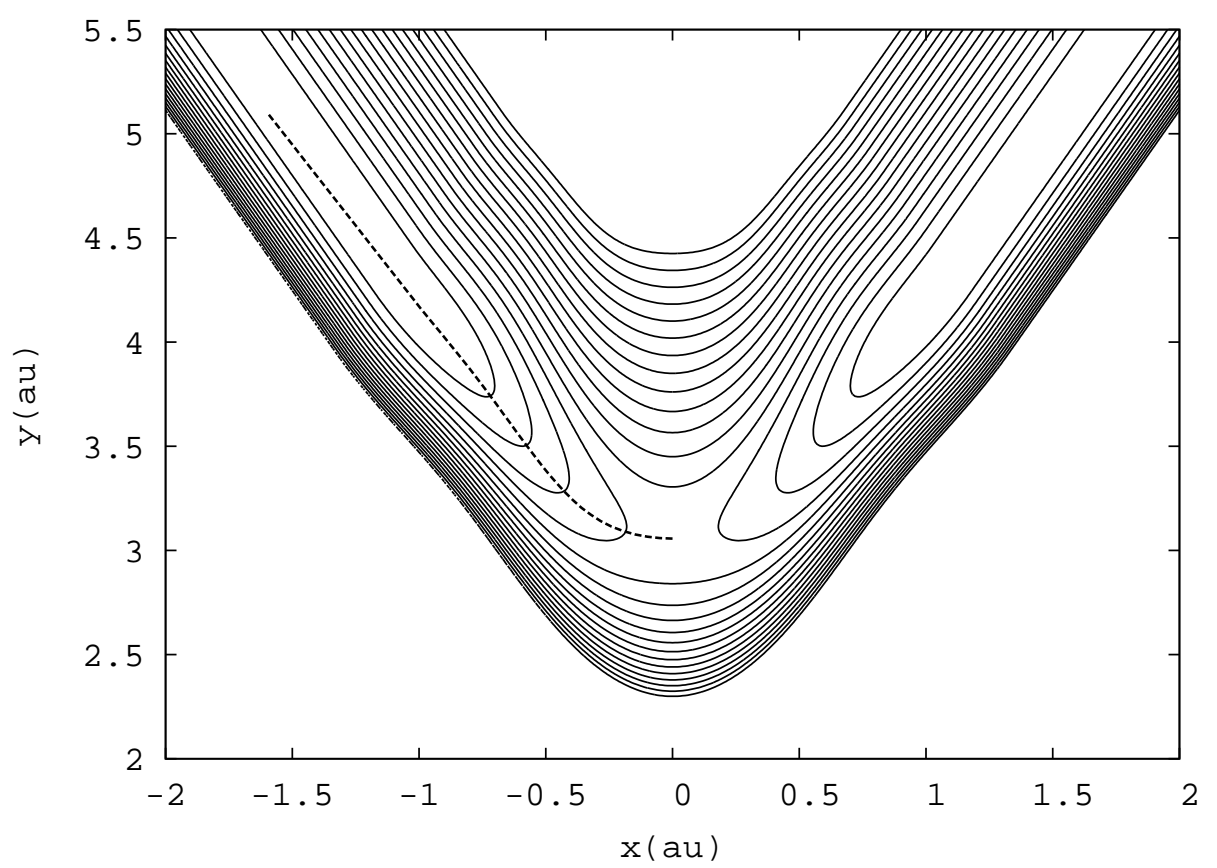

Figure 3: PES of the collinear $H+H_{2}$ reaction. The dashed line shows the MEP on the reactant side of the saddle point.

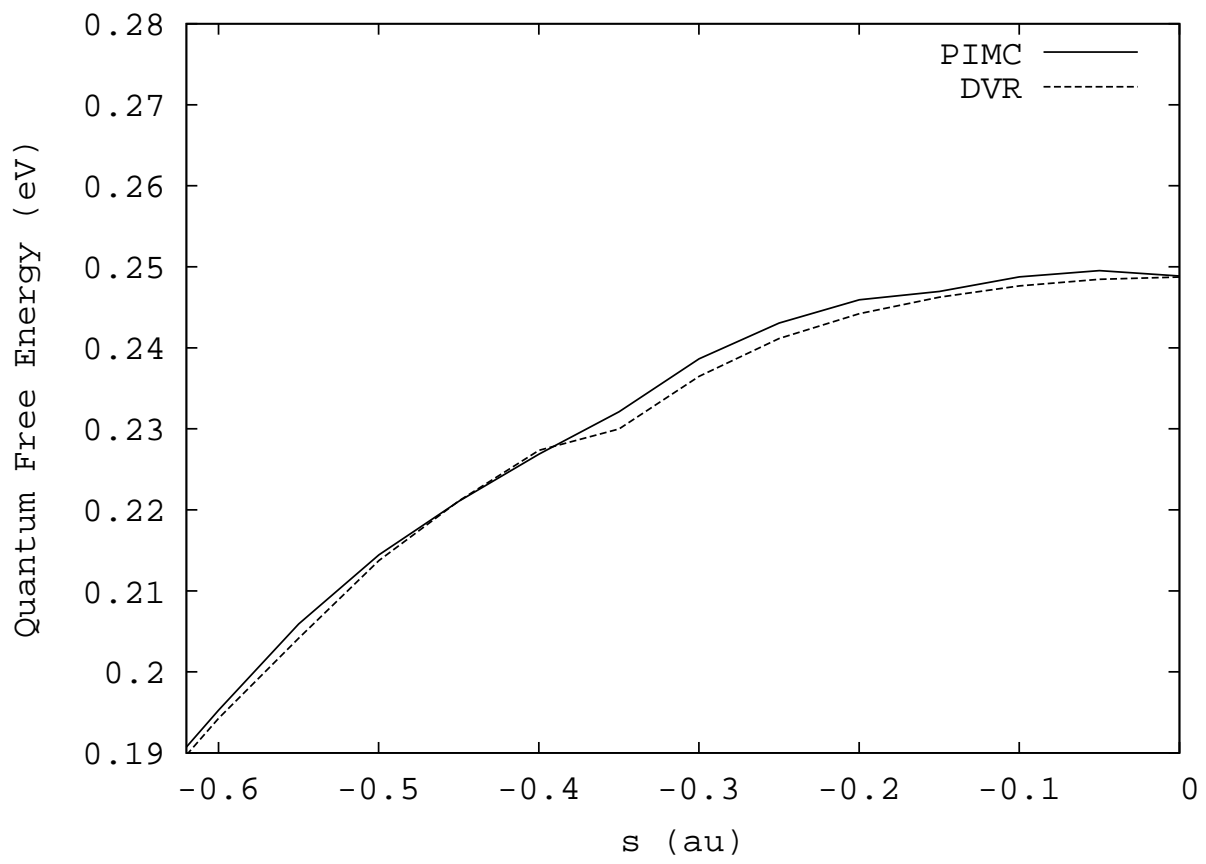

Figure 4: Quantum free energy for the collinear $\mathrm{H}+\mathrm{H}_{2}$ reaction. 


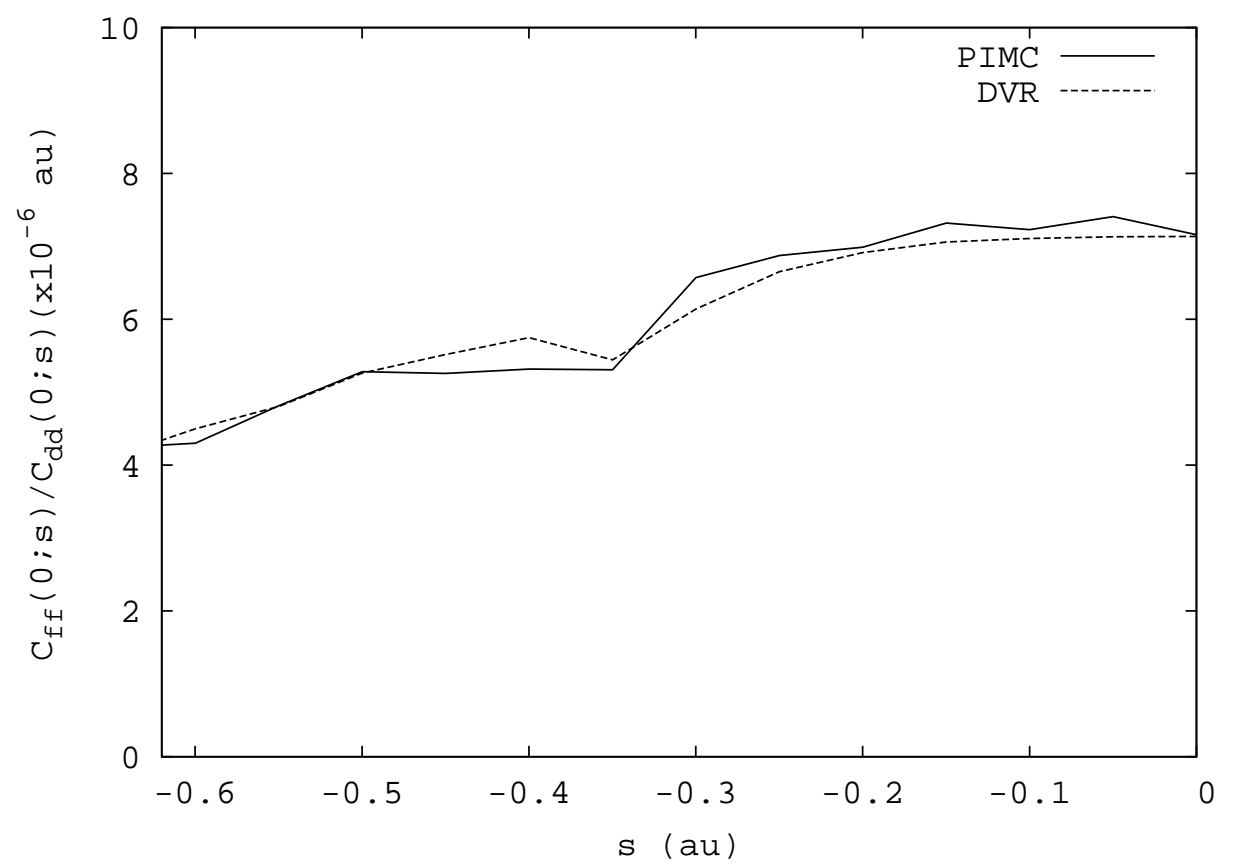

Figure 5: $C_{f f}(0 ; s) / C_{d d}(0 ; s)$ for the collinear $H+H_{2}$ reaction.

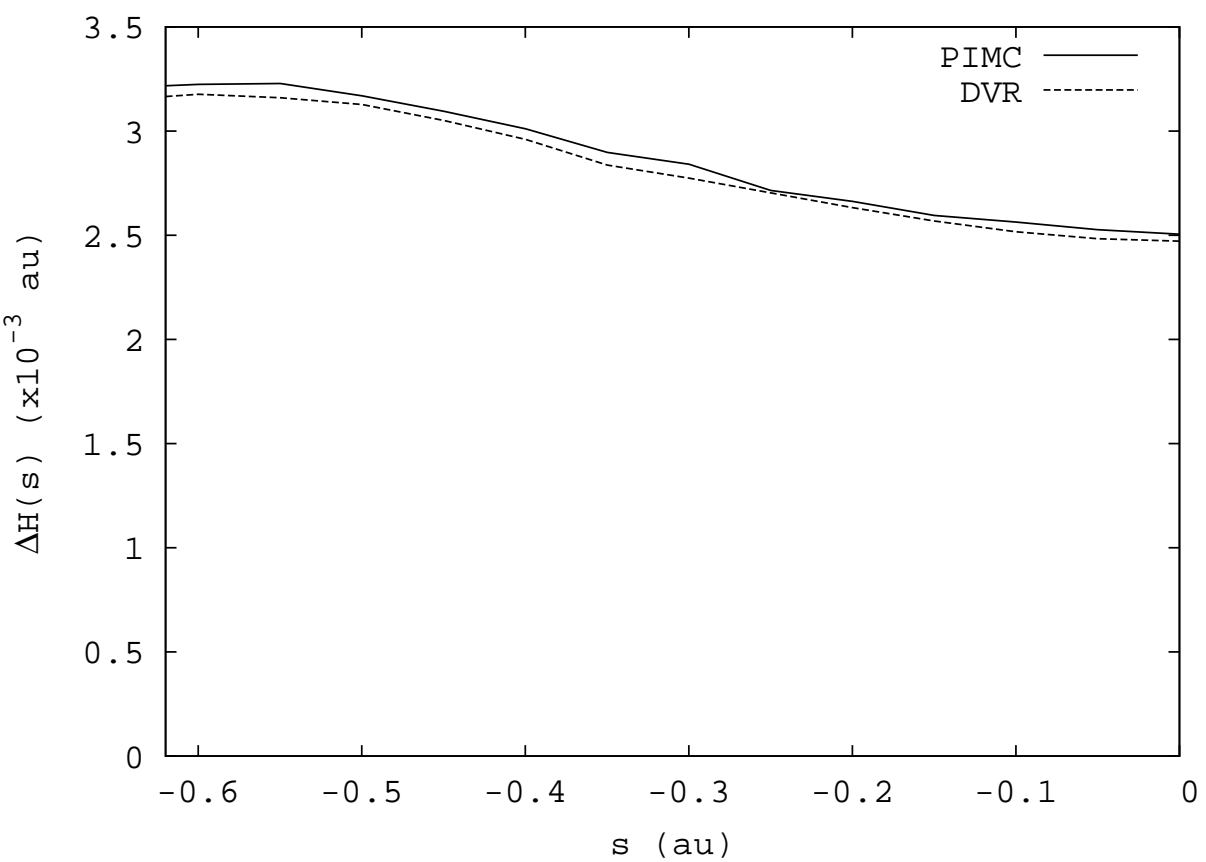

Figure 6: $\Delta H(s)$ for the collinear $H+H_{2}$ reaction. 


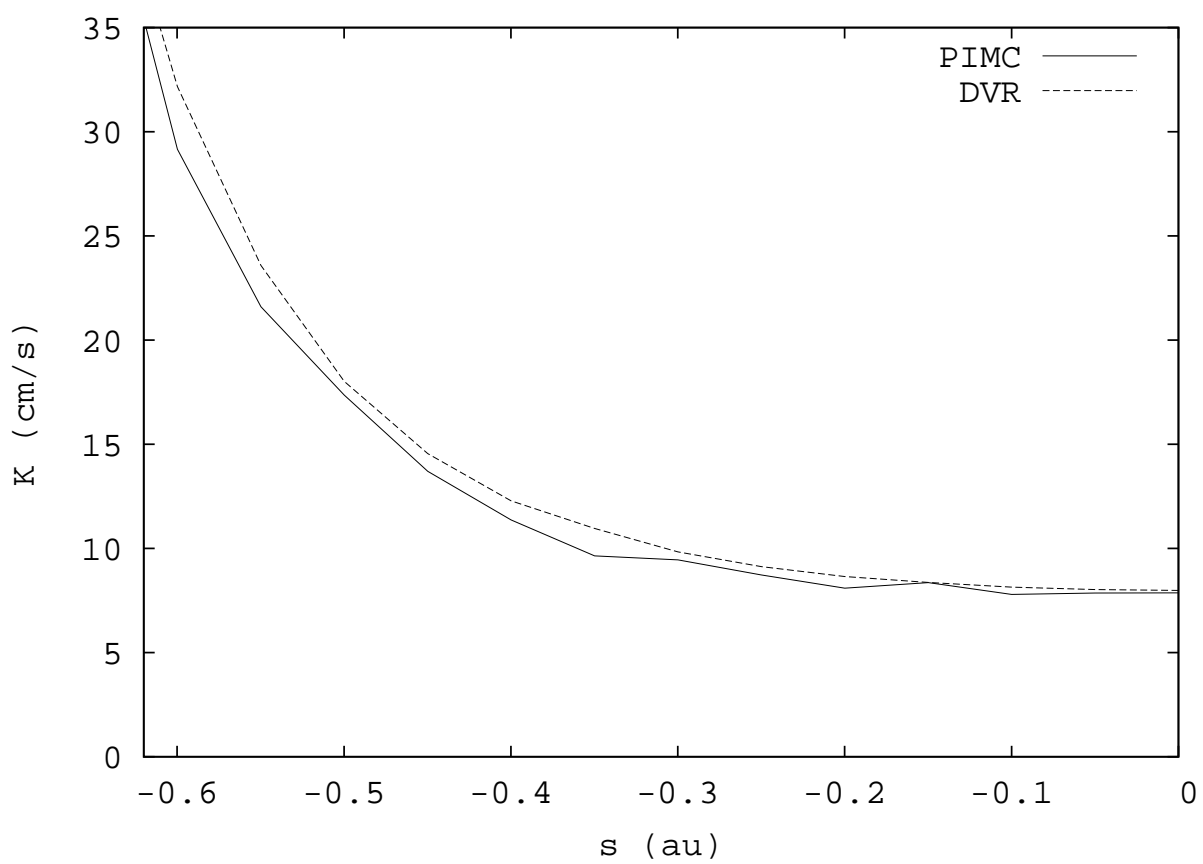

Figure 7: The QI thermal rate constant for the collinear $H+H_{2}$ reaction as a function of the position of DS. 